\title{
Consensus of High-Order Nonlinear Multiagent Systems with Constrained Switching Topologies
}

\author{
Junwei Wang, ${ }^{1}$ Kairui Chen, ${ }^{2}$ and Yun Zhang ${ }^{2}$ \\ ${ }^{1}$ Department of Applied Mathematics, Guangdong University of Foreign Studies, Guangzhou 510006, China \\ ${ }^{2}$ School of Automation, Guangdong University of Technology, Guangzhou 510006, China \\ Correspondence should be addressed to Junwei Wang; wangjwlj@gmail.com
}

Received 12 July 2016; Accepted 24 October 2016; Published 11 January 2017

Academic Editor: Pietro De Lellis

Copyright (c) 2017 Junwei Wang et al. This is an open access article distributed under the Creative Commons Attribution License, which permits unrestricted use, distribution, and reproduction in any medium, provided the original work is properly cited.

\begin{abstract}
The relationship between control and communication constraints is becoming of central importance in the consensus problem of networked agents. In this paper, we investigate such a problem for nonlinear multiagent systems with Lipschitz dynamics. To reflect communication constraints, the topology is assumed to switch within a finite set of digraphs characterised by an average dwell time switching signal. By constructing a suitable multiple Lyapunov function, we show that consensus can be reached under the designed consensus protocol. A multistep algorithm for designing consensus protocol is then developed by solving the Lyapunov equation and algebraic Riccati equation. Finally, simulation examples are delineated to substantiate the effectiveness of the proposed algorithms.
\end{abstract}

\section{Introduction}

The traditional study of single-agent systems has been overcome by a more realistic approach, that is, multiagent systems (MASs), which accounts for communications between agents. Over the past years, cooperative control of MASs has been a popular subject in systems and control community due to its broad applications in multirobot search and rescue, formation control of unmanned air vehicles, scheduling of automated highway systems, and attitude alignment of satellite clusters [1-3]. A fundamental issue for multiagent coordination is to design distributed control strategies guaranteeing that all agents reach an agreement about some variables of interest (such as position, velocity, and voltage), which is known as the consensus problem in the literature.

From the seminal work of $[4,5]$, the topic of consensus in a team of autonomous agents has rapidly grown and attracted the attention of many researchers; see, for example, [6-9] for first-order MASs, [10-13] for second-order MASs, [14] for high-order MASs, and the recent survey papers [15]. Under a static communication topology, it has been shown that the first-order MASs achieve consensus if and only if the directed topology contains a spanning tree or the undirected topology is connected [7]. Moreover, consensus under a dynamic topology can also be achieved in such systems as long as the union of the digraphs has a spanning tree or the union of the undirected graphs is connected frequently enough as the system evolves $[4,6,7]$. It is worth pointing out that, different from the first-order case, both communication topology and coupling strength will affect the information consensus in MASs with second-order or high-order integrator dynamics [10, 14]. However, most of these works usually assume that agents do not have their own dynamics in the absence of communication, indicating that only the information exchange determines agents' time evolution. Although this assumption makes consensus analysis much easier, it is too restrictive for many applications. In fact, for consensus problems encountered in the engineering world, both individual dynamics and interaction topology play an important role in achieving group consensus $[16,17]$.

In recent years, efforts have been made in generalizing existing results to MASs with general linear dynamics [1622]. For linear MASs under time-invariant topology, the distributed control laws based on state feedback or output 
feedback have been designed under which the systems will synchronize $[16,19]$. But some constraints were imposed on the system matrix of each individual agent; for example, the system matrix is required to be neutrally stable or have no eigenvalues with positive real parts. Also in the framework of fixed topology, $\mathrm{Ma}$ and Zhang [17] studied the joint impact of agent dynamics and communication topology on consensusability. When the underlying graphs are timevarying, leader-following consensus for multiple agents with general linear dynamics was discussed in [21] and [22] for switching undirected and directed topologies, respectively.

Compared with the development of general linear MASs, that for general nonlinear MASs is more limited with comparatively very few results available. Some works on consensus of nonlinear MASs with identical or heterogenous nonlinearity, for example, [23-25], required restrictive assumptions such as a fixed communication graph condition. However, this assumption may not be satisfied in reality due to technological limitations of sensors or external disturbances. A relevant but more general setting was studied in [26] which assumed the communication topology switches within a finite set of undirected graphs or digraphs. The recent works in [27-29] also discussed the consensus problem of general high-order nonlinear MASs in the leader-following or leadless framework. However, it should be pointed out that the LMI-type conditions for consensus in [26-29] not only are computationally time-consuming, but also obscure the relationship among consensus, agents' dynamics, and communication topology.

In this paper, we attempt to bridge the above theoretical gap by dealing with a fairly general solution of consensus problem in the nonlinear case. More specifically, with the underlying interaction topology switching within a finite set of digraphs, consensus of high-order nonlinear MASs is studied under the following conditions: (1) the intrinsic dynamics of each individual agent is in the Lipschitz nonlinear form, which is quite general and includes integrator agents and general linear agents in the consensus literature as special cases and (2) the communication topology of present MAS model is allowed to switch according to an average dwell time (ADT) switching signal. The idea of imposing constraints on the switching signal may arise naturally from physical constraints of mobile agents. Due to the introduction of ADT-type switching topology and nonlinear agent dynamics, the methods in existing literature concerning arbitrarily switching or dwell time switching topologies are not applicable. In this contribution, by constructing a multiple Lyapunov function, it is shown that the consensus problem can be solved under the proposed consensus protocol if the communication topology has a spanning tree and the ADT is larger than an explicitly calculated threshold. Our sufficient conditions suggest that reaching consensus in such systems is dependent not only on the communication topology but also on the agents' dynamics and switching topology. In addition, to fortify our contribution practically, we provide a specific multistep algorithm to determine the gain matrix of the proposed protocol relying on the algebraic graph theory, Lyapunov equation, and algebraic Riccati theory. We hope that our analysis will shed light on cooperative behavior emerging in complex MASs.

The remainder of the paper is organised as follows. Some background on graph theory as well as problem formulation is given in Section 2. In Section 3, we provide a preliminary consensus result on nonlinear MASs with fixed communication topology. Then we generalize that result to the restricted switching case and establish our main theorem in Section 4. The simulation examples are presented in Section 5 and followed by Section 6, which concludes this paper.

\section{Preliminaries and Problem Setup}

Throughout this paper, we let $\|\cdot\|$ denote the Euclidian norm. $I_{n}$ (or $O_{n}$ ) is the $n \times n$ identity matrix (or zero matrix); $1_{N}$ (or $0_{N}$ ) is a vector in $\mathbb{R}^{N}$ with elements being all ones (or all zeros). $\operatorname{diag}\left\{x_{1}, \ldots, x_{N}\right\}$ defines a diagonal matrix with diagonal entries $x_{1}$ to $x_{N} . \underline{N}=\{1,2, \ldots, N\}$ is an index set. We say $X>0$ (or $X<0$ ) if the matrix $X$ is positive (or negative) definite. If not explicitly stated, we assume the dimensions of the matrix used in this paper are compatible.

2.1. Preliminaries. In order to facilitate model description, we introduce some concepts and notions in algebraic graph theory [30]. Let $\mathscr{G}=(\mathscr{V}, \mathscr{E})$ denote a digraph with a set of nodes $\mathscr{V}=\{1, \ldots, N\}$ and a set of directed edges $\mathscr{E} \subseteq \mathscr{V} \times \mathscr{V}$ of the form $(i, j)$. Let us define a weighted adjacency matrix $\mathscr{A}=\left[a_{i j}\right] \in \mathbb{R}^{N \times N}$ as follows: $a_{i j}>0$ if $(j, i) \in \mathscr{E} ; a_{i j}=0$ otherwise. We assume there are no repeated edges and no self-loops; that is, $a_{i i}=0$ for all $i \in N$. If $(j, i) \in \mathscr{E}$, we say node $j$ is a neighbor of node $i$. The set of neighbors of node $i$ is denoted by $N_{i}=\{j \in \mathscr{V}:(j, i) \in \mathscr{E}\}$. A sequence of edges $\left(i_{1}, i_{2}\right),\left(i_{2}, i_{3}\right), \ldots,\left(i_{k-1}, i_{k}\right)$ is called a directed path from node $i_{1}$ to node $i_{k}$. A digraph is said to have a spanning tree if there exists a node, called the root, which has a directed path to every other node in the graph. The Laplacian matrix $L=\left[l_{i j}\right] \in \mathbb{R}^{N \times N}$ of graph $\mathscr{G}$ is defined by $L=\mathscr{D}-\mathscr{A}$, where $\mathscr{D}=\operatorname{diag}\left\{d_{1}, \ldots, d_{N}\right\}$ is the degree matrix with $d_{i}=\sum_{j \in N_{i}} a_{i j}$. An important property of $L$ is presented in the following lemma.

Lemma 1 (see [7]). If the digraph $\mathscr{G}$ has a spanning tree, then its Laplacian matrix $L$ has a simple zero eigenvalue and all other eigenvalues have positive real parts.

Let

$$
\begin{aligned}
& E=\left[-1_{N-1}, I_{N-1}\right] \in \mathbb{R}^{(N-1) \times N}, \\
& F=\left[0_{N-1}, I_{N-1}\right]^{T} \in \mathbb{R}^{N \times(N-1)} .
\end{aligned}
$$

By taking

$$
S=\left(\begin{array}{cc}
1 & 0_{N-1}^{T} \\
1_{N-1} & I_{N-1}
\end{array}\right),
$$

we have

$$
S^{-1} L S=\left(\begin{array}{cc}
0 & -\beta^{T} \\
0_{N-1} & E L F
\end{array}\right)
$$


where $\beta=\left(-l_{12},-l_{13}, \ldots,-l_{1 N}\right)^{T}$. Hence, from Lemma 1 , we can establish a key lemma on the eigenvalue distribution of matrix $E L F \in \mathbb{R}^{(N-1) \times(N-1)}$ as follows.

Lemma 2 (see [31]). If the digraph $\mathscr{G}$ has a spanning tree, then the eigenvalues of ELF correspond to the $N-1$ nonzero eigenvalues of Laplacian $L$ and all have positive real parts.

2.2. Problem Setup. In this paper, we consider a MAS consisting of $N$ nonlinear agents. Regarding $N$ agents as nodes in $\mathscr{V}$, the communication among them can be conveniently described by a digraph $\mathscr{G}=(\mathscr{V}, \mathscr{E})$, where $(j, i) \in \mathscr{E}$ represents that agent $i$ can receive information from agent $j$.

The dynamical behavior of each agent is described as

$$
\begin{array}{r}
\dot{x}_{i}(t)=A x_{i}(t)+f\left(x_{i}(t), t\right)+B u_{i}(t), \\
\quad i=1,2, \ldots, N,
\end{array}
$$

where $x_{i}(t)=\left(x_{i 1}, x_{i 2}, \ldots, x_{i n}\right)^{T} \in \mathbb{R}^{n}$ is the state of the $i$ th agent and $u_{i}(t) \in \mathbb{R}^{m}$ is the control protocol to be designed. $A \in \mathbb{R}^{n \times n}$ and $B \in \mathbb{R}^{n \times m}$ are constant real matrices. The pair $(A, B)$ is assumed to be stabilizable throughout the paper. The continuously differentiable function $f(\cdot, \cdot): \mathbb{R}^{n} \times \mathbb{R}^{+} \rightarrow$ $\mathbb{R}^{n}\left(f\left(x_{i}(t), t\right)=\left[f_{1}\left(x_{i}(t), t\right), \ldots, f_{n}\left(x_{i}(t), t\right)\right]^{T}\right)$ describes the intrinsic nonlinear dynamics of each agent and is assumed to satisfy the following Lipschitz condition.

Assumption 3. There exists a nonnegative constant $\eta$ such that for $\forall y, z \in \mathbb{R}^{n}$ and $\forall t \geq 0$

$$
\|f(y, t)-f(z, t)\| \leq \eta\|y-z\| .
$$

Remark 4. It is worth mentioning that the agent's dynamics in the MAS (4) is in a very general nonlinear form, which includes the first-order, second-order, and high-order integrators and general linear systems as special cases. Assumption 3 on $f(\cdot, \cdot)$ is very mild in the sense that it is automatically satisfied if $\partial f_{i} / \partial x_{j}(i, j=1,2, \ldots, n)$ are uniformly bounded. Therefore, it includes many well-known systems, such as various neural networks, Chua's oscillator, the Lorenz system, Chen system, and Lü system.

Here, the control objective of consensus regulation problem is to devise distributed algorithm $u_{i}(i \in \underline{N})$ such that all $N$ agents in (4) can reach a common state finally, which is precisely defined as follows.

Definition 5. The consensus of the MAS (4) is said to be achieved, if there is a distributed controller $u_{i}$ for each agent $i \in \underline{N}$ such that the closed-loop system satisfies

$$
\lim _{t \rightarrow \infty}\left\|x_{i}(t)-x_{j}(t)\right\|=0, \quad \forall i, j \in \underline{N}
$$

for any initial conditions $x_{i}(0)$ and $x_{j}(0)$.
In the following, by designing a distributed consensus protocol, we will demonstrate that the consensus of the MAS (4) depends on both the structure properties of each agent's dynamics and communication topology of the system.

\section{Consensus under Fixed Communication Topology}

In this section, we consider consensus of the MAS (4) with fixed communication topology. To solve the problem, we make the following assumption.

Assumption 6. The communication topology $\mathscr{G}$ contains a directed spanning tree.

We propose a distributed protocol of the form

$$
u_{i}=K \sum_{j \in N_{i}} a_{i j}\left(x_{j}(t)-x_{i}(t)\right), \quad i \in \underline{N},
$$

where $K \in \mathbb{R}^{m \times n}$ is a feedback gain matrix to be designed.

Define consensus error as $\delta_{i}(t) \triangleq x_{i}(t)-x_{1}(t)(i=$ $2, \ldots, N)$. From (4) and (7), we have the following error system:

$$
\begin{aligned}
\dot{\delta}_{i}(t)= & A \delta_{i}(t)+\left[f\left(x_{i}(t), t\right)-f\left(x_{1}(t), t\right)\right] \\
& +B K\left[\sum_{j=1}^{N}\left(a_{i j}-a_{1 j}\right) \delta_{j}(t)-d_{i} \delta_{i}(t)\right]
\end{aligned}
$$

which can then be expressed in a compact form as

$$
\dot{\delta}(t)=\left(I_{N-1} \otimes A-E L F \otimes B K\right) \delta(t)+\tilde{f}(x(t), t)
$$

by defining $\delta=\left(\delta_{2}^{T}, \delta_{3}^{T}, \ldots, \delta_{N}^{T}\right)^{T}$ and $\tilde{f}(x, t)=\left[f^{T}\left(x_{2}, t\right)-\right.$ $\left.f^{T}\left(x_{1}, t\right), \ldots, f^{T}\left(x_{N}, t\right)-f^{T}\left(x_{1}, t\right)\right]^{T}$. Here $E$ and $F$ are defined in (1).

Remark 7. From the definition of the consensus error $\delta_{i}(t)$, it can be seen that the consensus problem of the MAS (4) has been transformed to the asymptotical stability problem of error system (8) or (9). Moreover, we should note that, in $\delta_{i}(t) \triangleq x_{i}(t)-x_{1}(t)(i=2, \ldots, N), x_{1}(t)$ is selected as a reference state which is only convenient for the following stability analysis of the error system (8) or (9). As a matter of fact, other states $x_{2}(t), x_{3}(t), \ldots$, or $x_{N}(t)$ can also be chosen as the reference state without changing the present results.

Remark 8 . In this paper, the symbol $\otimes$ denotes Kronecker product (also known as the tensor product). For any matrices $A, B, C$, and $D$ with appropriate dimensions, $A \otimes B=\left[a_{i j} B\right]$ and the Kronecker algebra has the following properties [32]: $A \otimes(B+C)=A \otimes B+A \otimes C,(A \otimes B)^{T}=A^{T} \otimes B^{T}$, and $(A \otimes B)(C \otimes D)=A C \otimes B D$.

Under Assumption 6, it follows from Lemma 2 that all $N-1$ eigenvalues of $E L F$ in the error system (9) have positive real parts. Then, there exists a positive definite matrix $Q>0$ in $\mathbb{R}^{(N-1) \times(N-1)}$ satisfying the following Lyapunov equation:

$$
(E L F)^{T} Q+Q(E L F)=I_{N-1} .
$$


Denoting the $N-1$ positive real eigenvalue of $Q$ are $\lambda_{j}(Q)(j \in \underline{N-1})$. As the set $\left\{\lambda_{j}(Q): j \in \underline{N-1}\right\}$ is finite, we define

$$
\begin{aligned}
& \lambda_{\max }(Q)=\max \left\{\lambda_{j}(Q): j \in \underline{N-1}\right\}, \\
& \lambda_{\min }(Q)=\min \left\{\lambda_{j}(Q): j \in \underline{N-1}\right\}
\end{aligned}
$$

which are all positive.

On the other hand, associated with $(A, B)$, from LQRbased optimal control theory [33], the following algebraic Riccati equation (ARE)

$$
A^{T} P+P A-\frac{1}{\lambda_{\max }(Q)} P B B^{T} P+c I_{n}=0
$$

has a unique positive definite solution $P>0$ for any positive real number $c$ which will be specified later. Denote the $n$ positive real eigenvalue of $P$ by $\mu_{j}(P)(j \in \underline{n})$. Define

$$
\begin{aligned}
& \mu_{\max }(P)=\max \left\{\mu_{j}(P): j \in \underline{n}\right\}, \\
& \mu_{\min }(P)=\min \left\{\mu_{j}(P): j \in \underline{n}\right\}
\end{aligned}
$$

which are all positive.

Now, with the above preparations, we are in a position to give our first main result for the MAS (4) under the fixed interaction topology.

Theorem 9. Suppose that Assumptions 3 and 6 hold. Let $Q>0$ and $P>0$ be the solutions of (10) and (12), respectively, and the gain matrix in (7) is designed as $K=B^{T} P$. Then, the multiagent system (4) can achieve consensus under protocol (7) if the following inequality holds:

$$
c>\frac{\lambda_{\max }(Q) \mu_{\max }^{2}(P)}{\lambda_{\min }(Q) \mu_{\min }(P)} \eta^{2}+\mu_{\max }(P) \text {, }
$$

where $c$ is a positive constant given in (12).

Proof. We construct a Lyapunov function candidate for the error system (9) as

$$
V(t)=\delta^{T}(t)(Q \otimes P) \delta(t),
$$

where $Q>0$ and $P>0$ are the solutions of (10) and (12), respectively. (9) is

The time derivative of $V(t)$ along the trajectories of system

$$
\begin{aligned}
\dot{V}(t)= & 2 \delta^{T}(t)(Q \otimes P) \dot{\delta}(t) \\
= & 2 \delta^{T}(t)(Q \otimes P)\left(I_{N-1} \otimes A-E L F \otimes B K\right) \delta(t) \\
& +2 \delta^{T}(t)(Q \otimes P) \tilde{f}(x(t), t) .
\end{aligned}
$$

Substituting $K=B^{T} P$ into (9) and then applying Assumption 3, we have

$$
\begin{aligned}
& \dot{V}(t)=\delta^{T}(t)\left[Q \otimes\left(A^{T} P+P A\right)\right. \\
& \left.-\left((E L F)^{T} Q+Q(E L F)\right) \otimes P B B^{T} P\right] \delta(t)+2 \delta^{T}(t) \\
& \cdot(Q \otimes P) \tilde{f}(x(t), t) \leq \delta^{T}(t)\left[Q \otimes\left(A^{T} P+P A\right)\right. \\
& \left.-\frac{1}{\lambda_{\max }(Q)} Q \otimes P B B^{T} P\right] \delta(t)+2 \delta^{T}(t)(Q \otimes P) \\
& \cdot \tilde{f}(x(t), t)=\delta^{T}(t)[Q \\
& \left.\otimes\left(A^{T} P+P A-\frac{1}{\lambda_{\max }(Q)} P B B^{T} P\right)\right] \delta(t)+2 \delta^{T}(t) \\
& \cdot(Q \otimes P) \tilde{f}(x(t), t)=-\delta^{T}(t)\left[Q \otimes c I_{n}\right] \delta(t) \\
& +2 \delta^{T}(t)(Q \otimes P) \tilde{f}(x(t), t) \leq-\frac{c}{\mu_{\max }(P)} \delta^{T}(t)[Q \\
& \otimes P] \delta(t)+\tilde{f}^{T}(x(t), t)(Q \otimes P) \tilde{f}(x(t), t) \\
& +\delta^{T}(t)(Q \otimes P) \delta(t) \leq-\left(\frac{c}{\mu_{\max }(P)}\right. \\
& \left.-\frac{\lambda_{\max }(Q) \mu_{\max }(P)}{\lambda_{\min }(Q) \mu_{\min }(P)} \eta^{2}-1\right) \delta^{T}(t)[Q \otimes P] \delta(t) .
\end{aligned}
$$

By using the inequality (14), we have $\dot{V}(t)<0$ for any $\delta(t) \neq$ 0 . According to Lyapunov stability theory, one can conclude that the nonlinear error system (9) is asymptotically stable. Therefore, the consensus problem of the MAS (4) is solved by the distributed protocol (7). This completes the proof.

According to the proof of Theorem 9, a three-step procedure can be given to determine a consensus protocol (7) under a time-invariant topology containing a directed spanning tree.

\section{Algorithm 10.}

Step 1. Solve the Lyapunov equation (10) to get a matrix $Q>0$ and calculate its largest eigenvalue $\lambda_{\max }(Q)$.

Step 2. Choose an appropriate number $c>0$ and solve the Riccati equation (12) to get a matrix $P>0$. Then, design $K=$ $B^{T} P$.

Step 3. Verify that inequality (14) is feasible.

Remark 11. From the proof of Theorem 9, it can be seen that, by introducing a model transformation, the consensus problem of a large-scale multiagent system (4) with protocol (7) is converted into the stability problem of the error system (9) with a reduced-order dimension. The results show that both interaction topology (indicated by the Lyapunov equation 
(10)) and agent's dynamics (indicated by the Riccati equation (12) and the algebraic inequality (14)) play important roles in the consensus of the system.

Remark 12. To our knowledge, the works of [23, 27] also focused on global consensus of Lipschitz nonlinear MASs under time-invariant topology. It is worth pointing out that Theorem 9 is different from these existing results in at least three aspects. First, the communication topology in Theorem 9 is assumed to be directed and only required to contain a spanning tree. Such an assumption is weaker in contrast to that in [23] (where it is assumed to be bidirectional and connected). Second, here we discuss the consensus regulation problem which is different from the consensus tracking problem in [27]. Third, all conditions for consensus in $[23,27]$ need to check the solvability of LMIs. By contrast, the results given here only require checking the feasibility of an algebraic inequality (14).

For information consensus problem, a recent interesting topic is addressing the consensus of linear MASs $[16,17,19]$. Here, Theorem 9 can be also adapted to the consensus of such systems with $f\left(x_{i}(t), t\right)$ in (4) removed; that is,

$$
\dot{x}_{i}(t)=A x_{i}(t)+B u_{i}(t), \quad i=1,2, \ldots, N .
$$

The proof is based on an analogous version of Theorem 9 and hence the corresponding result is only summarized in the following corollary.

Corollary 13. Suppose that Assumption 6 holds. Let $Q>0$ and $P>0$ be the solutions of (10) and (12) for any $c>0$, respectively. Then, the linear multiagent system (18) can achieve consensus under protocol (7) by designing the feedback gain matrix as $K=$ $B^{T} P$.

Remark 14. Different from the integrator agents, the agent's dynamics of the linear multiagent systems will affect the consensus behavior. In the existing works, it is often required that matrix $A$ is neutrally stable [19] or all eigenvalues of $A$ sit in the closed left-half complex plane [16]. Different from them, this assumption is now weakened to stabilizability of the pair $(A, B)$, which means that matrix $A$ can be stable, marginally stable, or even unstable.

\section{Consensus under Constrained Switching Topology}

In this section, we are interested in extending the results in Section 3 to the case that the interaction topology is switching according to a constrained switching signal. Associated with this case, the Laplacian matrix $L$ and the neighbor $N_{i}$ of each agent will vary with time $t$. Accordingly, we can define a switching digraph $\mathscr{G}_{\sigma(t)}=\left(\mathscr{V}, \mathscr{E}_{\sigma(t)}\right)$ to describe the communication relationship of multiagent system (4) at time $t$, where $\mathscr{E}_{\sigma(t)}$ denotes the directed edge set at time $t$. Let $\mathscr{A}_{\sigma(t)}=\left[a_{i j}^{\sigma(t)}\right], \mathscr{L}_{\sigma(t)}$, and $N_{i}^{\sigma(t)}$ be the weighted adjacency matrix, Laplacian matrix, and neighbor set of the switching graph, respectively. $\sigma(t):[0,+\infty) \rightarrow \mathscr{M}$ is a piecewise constant function, called a switching signal, which takes its values in the finite set $\mathscr{M}=\{1,2, \ldots, M\}$. Also, for any switching sequence $0=t_{0}<t_{1}<\cdots<t_{k}<t_{k+1}<\cdots$, $\sigma(t)$ is everywhere continuous from the right. Let $\sigma\left(t_{k}\right)=i_{k}$ for $i_{k} \in \mathscr{M}$, which means that the $i_{k}$ th interaction topology $\mathscr{G}_{i_{k}}$ is active when $t \in\left[t_{k}, t_{k+1}\right)$. We denote the finite set of all possible $M$ digraphs defined on the node set $\mathscr{V}$ as $\overline{\mathscr{G}}=\left\{\mathscr{G}_{1}, \mathscr{G}_{2}, \ldots, \mathscr{G}_{M}\right\}$. The following assumption on $\overline{\mathscr{G}}$ is a key condition to ensure consensus under switching topology.

Assumption 15. Each communication topology $\mathscr{G}_{m}$ in $\overline{\mathscr{G}}$ contains a directed spanning tree.

For the purpose of consensus under switching topology, we slightly modify the earlier protocol (7) to include a switching signal $\sigma(t)$. Thus, in the sequel we consider protocols of the form

$$
u_{i}=K \sum_{j \in N_{i}^{\sigma(t)}} a_{i j}^{\sigma(t)}\left(x_{j}(t)-x_{i}(t)\right), \quad i \in \underline{N},
$$

where $K \in \mathbb{R}^{m \times n}$ is the feedback gain matrix to be designed later.

Combining (19) with (4) leads to dynamics of the error system under switching topology

$$
\begin{aligned}
\dot{\delta}(t)= & \left(I_{N-1} \otimes A-E L_{\sigma(t)} F \otimes B K\right) \delta(t) \\
& +\widetilde{f}(x(t), t),
\end{aligned}
$$

where $E$ and $F$ are defined in (1).

As before, for each $m \in \mathscr{M}$, consider the Lyapunov equation

$$
\left(E L_{m} F\right)^{T} Q_{m}+Q_{m}\left(E L_{m} F\right)=I_{N-1}, \quad m \in \mathscr{M} .
$$

Under Assumption 15, (21) has a unique positive definite solution $Q_{m}>0$. Then, within the set of $M(N-1)$ positive eigenvalues $\left\{\lambda_{j}\left(Q_{m}\right): m \in \mathscr{M}, j \in \underline{N-1}\right\}$, we let

$$
\begin{aligned}
& \lambda_{\text {max }}=\max \left\{\lambda_{j}\left(Q_{m}\right): m \in \mathscr{M}, j \in \underline{N-1}\right\}, \\
& \lambda_{\text {min }}=\min \left\{\lambda_{j}\left(Q_{m}\right): m \in \mathscr{M}, j \in \underline{N-1}\right\} .
\end{aligned}
$$

On the other hand, since $(A, B)$ is stabilizable, from LQRbased optimal control theory [33], the following algebraic Riccati equation (ARE)

$$
A^{T} P+P A-\frac{1}{\lambda_{\max }} P B B^{T} P+c I_{n}=0
$$

has a unique positive definite solution $P>0$ for any positive real number $c$ which will be specified later. Within the set of $n$ positive eigenvalues $\left\{\mu_{j}(P): j \in \underline{n}\right\}$ of $P$, we let

$$
\begin{aligned}
& \mu_{\max }(P)=\max \left\{\mu_{j}(P): j \in \underline{n}\right\}, \\
& \mu_{\text {min }}(P)=\min \left\{\mu_{j}(P): j \in \underline{n}\right\} .
\end{aligned}
$$

Here, we aim at finding a general set of ADT switching signals and the corresponding state feedback controllers, such that the multiagent system (4) achieves consensus under protocol (19). For this purpose, let us first revisit the definition of the ADT property. 
Definition 16 (see [34]). For a switching signal $\sigma(t)$ and any $T_{2}>T_{1} \geq 0$, let $N_{\sigma}\left(T_{1}, T_{2}\right)$ denote the switching number of $\sigma(t)$ over the interval $\left(T_{1}, T_{2}\right)$. If there exist a positive number $\tau_{a}>0$ and an integer $N_{0} \geq 0$ such that

$$
N_{\sigma}\left(T_{1}, T_{2}\right) \leq N_{0}+\frac{T_{2}-T_{1}}{\tau_{a}},
$$

then $\tau_{a}$ is called an average dwell time.

Remark 17. Definition 16 means that if there exists a positive number $\tau_{a}$ such that a switching signal has the ADT property, the ADT between any two consecutive switching instants is no smaller than a common constant $\tau_{a}$ for all system modes.

Now, the following theorem states our second main result for the MAS (4) under the ADT switching topology.

Theorem 18. Suppose that Assumptions 3 and 15 hold. Let $Q_{m}>0$ and $P>0$ be the solutions of (21) and (23), respectively, and the gain matrix in (19) is designed as $K=B^{T} P$. Then, the multiagent system (4) can achieve consensus under protocol (19) if the positive constant $c$ in (23) satisfies

$$
\alpha \triangleq \frac{c}{\mu_{\max }(P)}-\frac{\lambda_{\max } \mu_{\max }(P)}{\lambda_{\min } \mu_{\min }(P)} \eta^{2}-1>0,
$$

and the ADT of switching signal $\sigma(t)$ satisfies

$$
\tau_{a}>\tau_{a}^{*}=\frac{\ln \beta}{\alpha},
$$

where $\beta \geq 1$ is chosen such that

$$
Q_{l} \leq \beta Q_{m}, \quad \forall l, m \in \mathscr{M} .
$$

Proof. Referring to the proof of Theorem 9, we see that it is equivalent to prove the asymptotical stability of error system (20) which is a nonlinear switched system.

This proof consists of two parts.

First, we consider the $m$ th subsystem of the nonlinear switched system (20)

$$
\begin{array}{r}
\dot{\delta}(t)=\left(I_{N-1} \otimes A-E L_{m} F \otimes B K\right) \delta(t)+\tilde{f}(x(t), t), \\
\forall m \in \mathscr{M}
\end{array}
$$

for which we choose the following Lyapunov function candidate

$$
V_{m}(t)=\delta^{T}(t)\left(Q_{m} \otimes P\right) \delta(t),
$$

where $Q_{m}>0$ and $P>0$ are the solutions of (21) and (23), respectively.

Substituting $K=B^{T} P$ into (20) and differentiating $V_{m}(t)$ along the trajectory of (20), we have

$$
\begin{aligned}
& \dot{V}_{m}(t)=\delta^{T}(t)\left[Q_{m} \otimes\left(A^{T} P+P A\right)\right. \\
& \left.-\left(\left(E L_{m} F\right)^{T} Q_{m}+Q_{m}\left(E L_{m} F\right)\right) \otimes P B B^{T} P\right] \delta(t) \\
& +2 \delta^{T}(t)\left(Q_{m} \otimes P\right) \tilde{f}(x(t), t) \leq \delta^{T}(t)\left[Q_{m}\right. \\
& \left.\otimes\left(A^{T} P+P A\right)-\frac{1}{\lambda_{\max }} Q_{m} \otimes P B B^{T} P\right] \delta(t)
\end{aligned}
$$

$$
\begin{aligned}
& +2 \delta^{T}(t)\left(Q_{m} \otimes P\right) \tilde{f}(x(t), t)=-\delta^{T}(t)\left[Q_{m} \otimes c I_{n}\right] \\
& \cdot \delta(t)+2 \delta^{T}(t)\left(Q_{m} \otimes P\right) \tilde{f}(x(t), t) \leq-\frac{c}{\mu_{\max }(P)} \\
& \cdot \delta^{T}(t)\left[Q_{m} \otimes P\right] \delta(t)+\tilde{f}^{T}(x(t), t)\left(Q_{m} \otimes P\right) \\
& \cdot \tilde{f}(x(t), t)+\delta^{T}(t)\left(Q_{m} \otimes P\right) \delta(t) \leq-\left(\frac{c}{\mu_{\max }(P)}\right. \\
& \left.-\frac{\lambda_{\max } \mu_{\max }(P)}{\lambda_{\min } \mu_{\min }(P)} \eta^{2}-1\right) \delta^{T}(t)\left[Q_{m} \otimes P\right] \delta(t) \\
& =-\alpha V_{m}(t),
\end{aligned}
$$

which is obtained by applying Assumption 3.

Integrating the above inequality on the interval $\left[t_{k}, t\right)$ gives

$$
V_{m}(t) \leq e^{-\alpha\left(t-t_{k}\right)} V_{m}\left(t_{k}\right), \quad \forall m \in \mathscr{M} .
$$

Second, for the error system (20), choose the following switched Lyapunov function

$$
V_{\sigma(t)}(t)=\delta^{T}(t)\left(Q_{\sigma(t)} \otimes P\right) \delta(t) .
$$

Let $0=t_{0}<t_{1}<\cdots<t_{k}<t_{k+1}<\cdots$ be any switching sequence of $\sigma(t)$. Then, at the switching instant $t_{k}$, combining (28) and (33) yields

$$
V_{\sigma\left(t_{k}\right)}\left(t_{k}\right) \leq \beta V_{\sigma\left(t_{k}^{-}\right)}\left(t_{k}^{-}\right), \quad k=1,2, \ldots
$$

Therefore, when $t \in\left[t_{k}, t_{k+1}\right)$, it follows from (32) and (34) that

$$
\begin{aligned}
V_{\sigma(t)}(t) & \leq e^{-\alpha\left(t-t_{k}\right)} V_{\sigma\left(t_{k}\right)}\left(t_{k}\right) \leq e^{-\alpha\left(t-t_{k}\right)} \beta V_{\sigma\left(t_{k}^{-}\right)}\left(t_{k}^{-}\right) \\
& \leq e^{-\alpha\left(t-t_{k}\right)} \beta e^{-\alpha\left(t_{k}-t_{k-1}\right)} V_{\sigma\left(t_{k-1}\right)}\left(t_{k-1}\right) \\
& \leq e^{-\alpha\left(t-t_{k}\right)} \beta^{2} e^{-\alpha\left(t_{k}-t_{k-1}\right)} V_{\sigma\left(t_{k-1}^{-}\right)}\left(t_{k-1}^{-}\right) \leq \cdots \\
& \leq e^{-\alpha\left(t-t_{0}\right)} \beta^{k} V_{\sigma\left(t_{0}\right)}\left(t_{0}\right) \\
& =e^{-\alpha\left(t-t_{0}\right)+N_{\sigma}\left(t_{0}, t\right) \ln \beta} V_{\sigma\left(t_{0}\right)}\left(t_{0}\right) \\
& \leq \beta^{N_{0}} e^{-\left(\alpha-\ln \beta / \tau_{a}\right)\left(t-t_{0}\right)} V_{\sigma\left(t_{0}\right)}\left(t_{0}\right)
\end{aligned}
$$

where (35) is obtained by applying (25) with $N_{\sigma}\left(t_{0}, t\right)=k$.

Combining (35) with $\lambda_{\min } \mu_{\min }(P)\|\delta(t)\|^{2} \leq V_{\sigma(t)}(t)$, we have

$$
\begin{aligned}
\|\delta(t)\|^{2} & \leq \frac{1}{\lambda_{\min } \mu_{\min }(P)} V_{\sigma(t)}(t) \\
& \leq \frac{\lambda_{\max } \mu_{\max }(P)}{\lambda_{\min } \mu_{\min }(P)} \beta^{N_{0}} e^{-\left(\alpha-\ln \beta / \tau_{a}\right)\left(t-t_{0}\right)}\left\|\delta\left(t_{0}\right)\right\|^{2}
\end{aligned}
$$

which means that

$\|\delta(t)\|$

$$
\leq \sqrt{\frac{\lambda_{\max } \mu_{\max }(P)}{\lambda_{\min } \mu_{\min }(P)}} \beta^{N_{0} / 2} e^{-(1 / 2)\left(\alpha-\ln \beta / \tau_{a}\right)\left(t-t_{0}\right)}\left\|\delta\left(t_{0}\right)\right\| .
$$


Clearly, if conditions (26) and (27) hold, we can conclude that $\|\delta(t)\| \rightarrow 0$ asymptotically, which implies that consensus problem in multiagent system (4) is solved by protocol (19) under the ADT switching topology. This completes the proof.

Based on the proof process of Theorem 18, a three-step algorithm is provided to determine a consensus protocol (19) under a time-varying topology.

\section{Algorithm 19.}

Step 1. For each possible digraph $\mathscr{G}_{m}$, solve the Lyapunov equation (21) to get a matrix $Q_{m}>0$ and calculate its largest eigenvalue $\lambda_{\max }$ among all $M$ digraphs.

Step 2. Choose an appropriate number $c>0$ and solve the Riccati equation (23) to get a matrix $P>0$. Then, design $K=$ $B^{T} P$.

Step 3. Verify that inequalities (26)-(28) are feasible.

Remark 20. In related works [26-29], all the consensus conditions involve the feasibility of LMIs. Here, a unique feature of Algorithm 19 is that a positive scalar $c$ is introduced. With this parameter, consensus is preserved under ADT switching topology only if the algebraic inequality (26) is verified to be feasible. Moreover, the size of matrices in the Lyapunov equation (21) and Riccati equation (23) to be computed is irrelevant to the number of individual agents. Therefore, nonlinear MASs with considerable size are still computationally manageable.

As in Corollary 13, the results of Theorem 18 are easily adapted to deal with a general linear MAS (18) under ADT switching topology. The corresponding result is summarized in the following corollary.

Corollary 21. Suppose that Assumption 15 holds. Let $Q_{m}>0$ and $P>0$ be the solutions of (21) and (23) for any $c>$ 0 , respectively, and the gain matrix in (23) is designed as $K=B^{T} P$. Then, the linear multiagent system (18) can achieve consensus under protocol (19) if the ADT of switching signal $\sigma(t)$ satisfies

$$
\tau_{a}>\tau_{a}^{*}=\frac{\ln \beta}{\alpha},
$$

where $\beta \geq 1$ is chosen such that

$$
Q_{l} \leq \beta Q_{m}, \quad \forall l, m \in M .
$$

Remark 22. For consensus analysis, the agent matrix $A$ in [21] should satisfy the inequality $A^{T} P+P A \leq 0$, which is not needed in Corollary 21. This means that the requirement of the agent's dynamics here is weaker than that in [21].

\section{Numerical Simulation}

In this section, we present two numerical examples to illustrate the effectiveness of the theoretical results in previous sections.

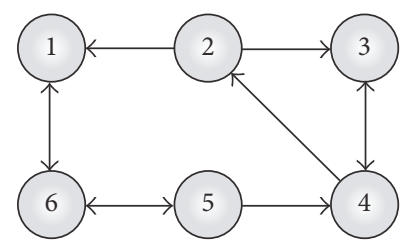

Figure 1: The directed interaction topology in Example 1.

Example 1 (consensus of multiple Chua's oscillators under fixed topology). To illustrate Theorem 9, we consider a MAS composed of six nonlinear agents with a fixed communication graph shown in Figure 1, in which the weights on directed edges are assumed to be 1. Each agent is modeled as a Chua's oscillator $[35,36]$ governed by (4) with

$$
\begin{aligned}
& A=\left(\begin{array}{rrr}
-\frac{2 m}{7} & m & 0 \\
1 & -1 & 1 \\
0 & -n & 0
\end{array}\right), \\
& B=15 I_{3},
\end{aligned}
$$

and $f\left(x_{i}\right)=\left((3 m / 14)\left(\left|x_{i 1}+1\right|-\left|x_{i 1}-1\right|\right), 0,0\right)^{T}$ where $x_{i}=\left(x_{i 1}, x_{i 2}, x_{i 3}\right)^{T}(i=1, \ldots, 6)$. It is known that Chua's circuit depicts a chaotic behavior when $m=5$ and $n=6(2 / 7)$. Apparently, the pair $(A, B)$ is stabilizable and $f\left(x_{i}\right)$ satisfies Assumption 3 with Lipschitz constant $\eta=3 m / 7$.

The control gain $K$ of the consensus protocol (7) is chosen based on the design procedure in Algorithm 10. Solving the Lyapunov equation (10) yields

$$
\begin{aligned}
& Q \\
& =\left(\begin{array}{ccccc}
0.4167 & 0.0042 & 0.0618 & -0.2500 & -0.1451 \\
0.0042 & 0.3392 & 0.1784 & 0.0069 & -0.1402 \\
0.0618 & 0.1784 & 0.3701 & -0.0174 & -0.1791 \\
-0.2500 & 0.0069 & -0.0174 & 0.5833 & 0.1007 \\
-0.1451 & -0.1402 & -0.1791 & 0.1007 & 0.3215
\end{array}\right) .
\end{aligned}
$$

A straightforward calculation shows that the largest eigenvalue of $Q$ is $\lambda_{\max }(Q)=0.87$ and the smallest eigenvalue of $Q$ is $\lambda_{\min }(Q)=0.14$. Then, solving the algebraic Riccati equation (12) with $c=6$, we obtain

$$
P=\left(\begin{array}{ccc}
0.1465 & 0.0111 & 0.0004 \\
0.0111 & 0.1504 & -0.0101 \\
0.0004 & -0.0101 & 0.1514
\end{array}\right),
$$

and thus design $K=B^{T} P$. Moreover, the selected parameter $c=6$ makes inequality (14) feasible. Figures 2 and 3 show the simulation results of applying consensus protocol (7) to the nonlinear MAS (4), from which we can see that the consensus behavior of general high-order MAS (4) under fixed directed topology is achieved. 


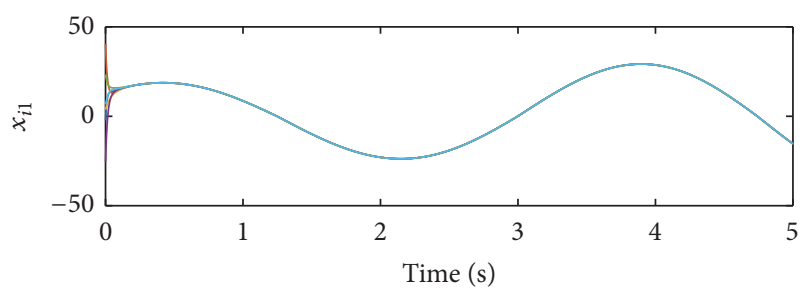

(a)

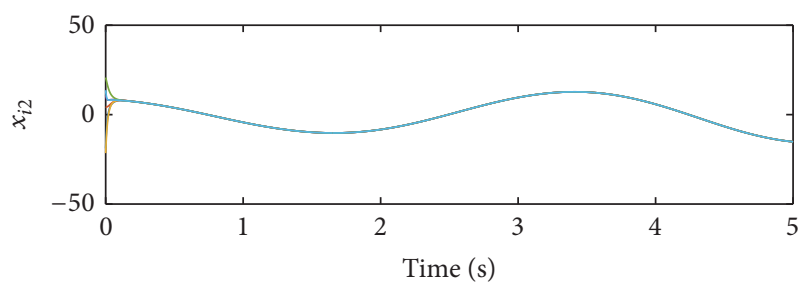

(b)

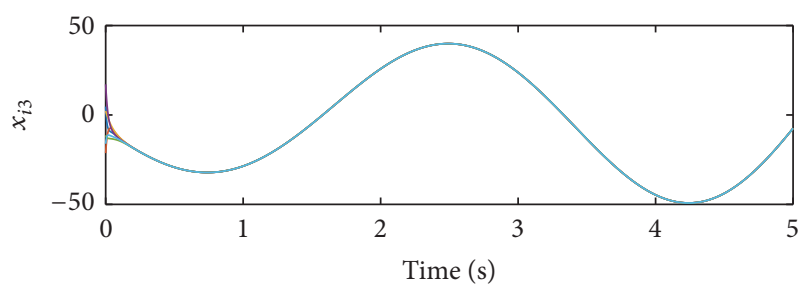

(c)

Figure 2: Time evolution of state trajectories $x_{i}(t)(i=1, \ldots, 6)$ in Example 1.

Example 2 (consensus of multiple single-link robots under restricted switching topology). To illustrate Theorem 18, we now employ a MAS composed of four single-link robots with revolute joints [37], whose dynamics can be written in the form of (4) with

$$
\begin{aligned}
A & =\left(\begin{array}{cccc}
0 & 10 & 0 & 0 \\
-48.6 & -1.25 & 48.6 & 0 \\
0 & 0 & 0 & 10 \\
19.5 & 0 & -19.5 & 0
\end{array}\right), \\
B & =\left(\begin{array}{c}
0 \\
21.6 \\
0 \\
0
\end{array}\right)
\end{aligned}
$$

and $f\left(x_{i}\right)=\left(0,0,0,-0.0333 \sin \left(x_{i 3}\right)\right)^{T}$, where $x_{i}=\left(x_{i 1}, x_{i 2}\right.$, $\left.x_{i 3}, x_{i 4}\right)^{T}(i=1, \ldots, 4)$. Here, the states $x_{i 1}$ and $x_{i 2}$ represent the angular rotation and angular velocity of the motor, respectively; $x_{i 3}$ and $x_{i 4}$ represent the angular rotation and angular velocity of the link, respectively. It is easy to check that the pair $(A, B)$ is stabilizable and the Lipschitz constant for this $f\left(x_{i}\right)$ is $\eta=0.0333$. The switching communication graph is shown in Figure 4, in which each possible graph contains a rooted spanning tree and the weights on directed edges are assumed to be 1 .

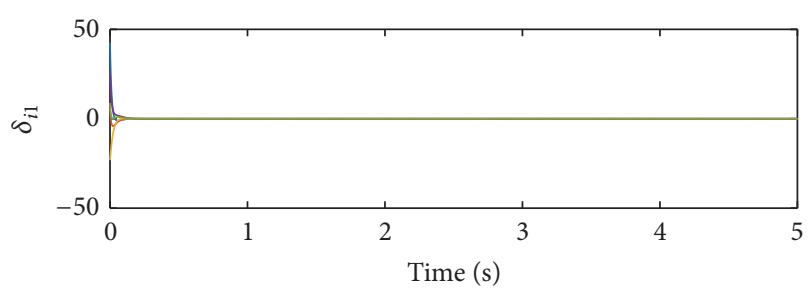

(a)

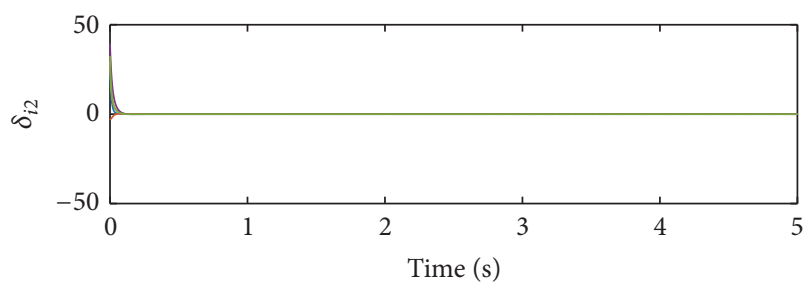

(b)

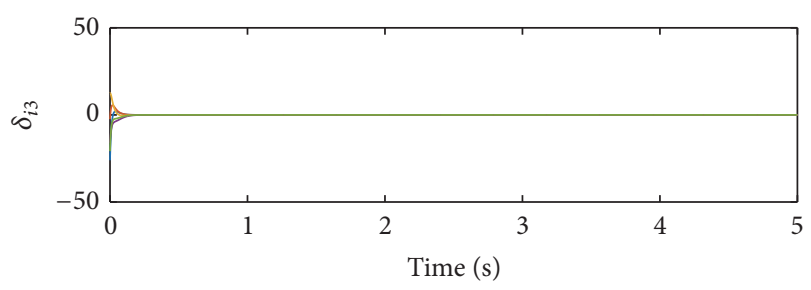

(c)

FIgURE 3: Time evolution of consensus errors $\delta_{i}(t)=x_{i}(t)-$ $x_{1}(t)(i=2, \ldots, 6)$ in Example 1.
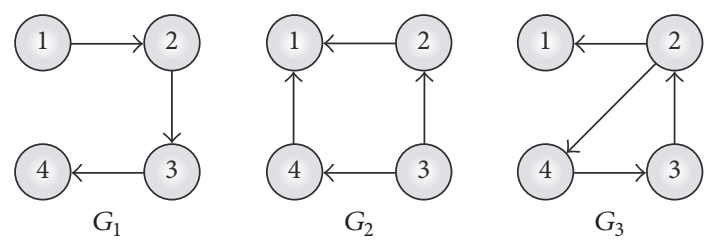

FIGURE 4: Three directed interaction topologies $G_{1}, G_{2}$, and $G_{3}$ in Example 2.

The control gain $K$ of the consensus protocol (19) is chosen based on the design procedure in Algorithm 19. For each directed graph $\mathscr{G}_{m}(m=1,2,3)$, we can get a positive matrix $Q_{m}$ by solving its corresponding Lyapunov equation (21). Due to the limited space, the details of $Q_{m}$ in Lyapunov equation (21) are omitted here. Then the largest and smallest eigenvalues of $Q_{1}, Q_{2}$, and $Q_{3}$ can be computed as $\lambda_{\text {max }}=1.37$ and $\lambda_{\min }=0.19$. Then, setting $c=41.5$ and solving the algebraic Riccati equation (23), we get

$$
P=\left(\begin{array}{cccc}
11.0675 & 0.7118 & -4.2657 & 5.1437 \\
0.7118 & 0.4005 & -0.2186 & 0.3571 \\
-4.2657 & -0.2186 & 10.2756 & 0.1008 \\
5.1437 & 0.3571 & 0.1008 & 7.5259
\end{array}\right)
$$

The feedback gain $K$ is designed as $K=B^{T} P$. With the above $c=41.5$, the algebraic inequality (26) is feasible with $\alpha=$ 


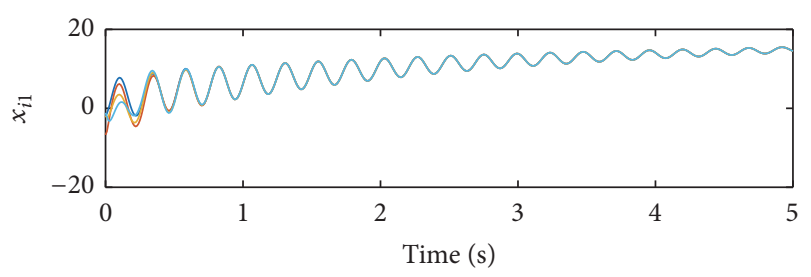

(a)

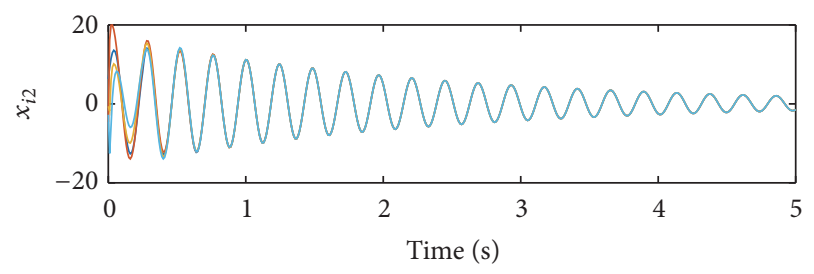

(b)

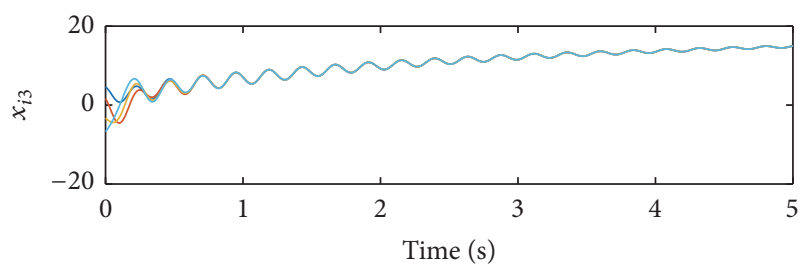

(c)

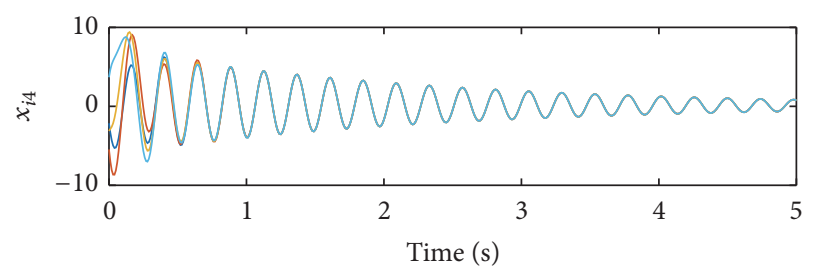

(d)

Figure 5: Time evolution of state trajectories $x_{i}(t)(i=1,2,3,4)$ in Example 2.

1.11. Then, we select the parameters $\beta$ in (28) as $\beta=6.49$. Thus, global consensus in the MAS (4) with protocol (19) will be achieved if the ADT $\tau_{a}$ of the switching signal $\sigma(t)$ is larger than $\tau_{a}^{*}=1.69$ which is obtained from (27).

With $\tau_{a}=1.8$ and $N_{0}=2$, Figures 5 and 6 show the state and the error trajectories for all the agents, respectively. It is observed that cooperative consensus is well achieved in spite of the fact that some dwell time of the switching signal $\sigma(t)$ between consecutive switching signals is smaller than the common constant $\tau_{a}$, as shown in Figure 7.

\section{Conclusions}

In this paper, we have studied the consensus problem for a class of MASs with Lipschitz nonlinear dynamics. A distinct feature of the MAS model lies in its communication topology that switches within a finite set of digraphs characterised by an ADT switching signal. The assumption not only reflects physical communication constraints of real mobile MASs, but also makes the methods in existing literature infeasible to

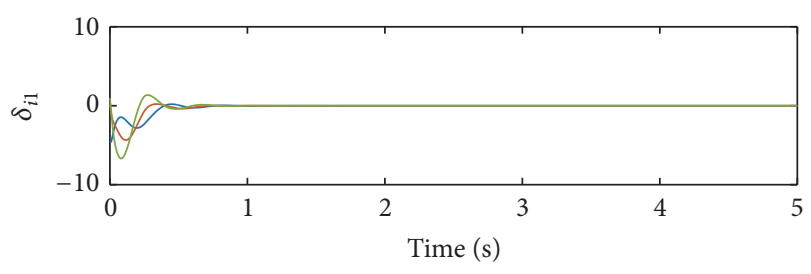

(a)

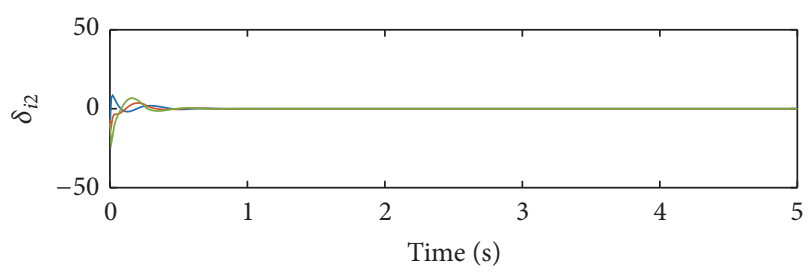

(b)

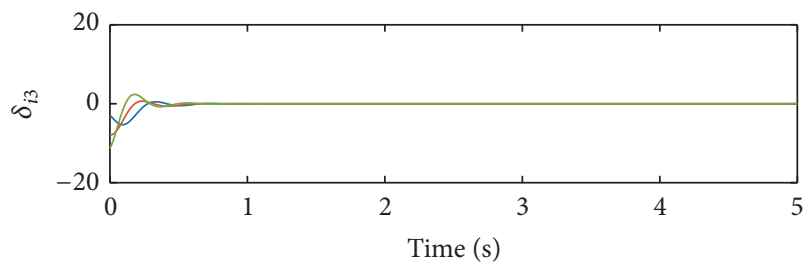

(c)

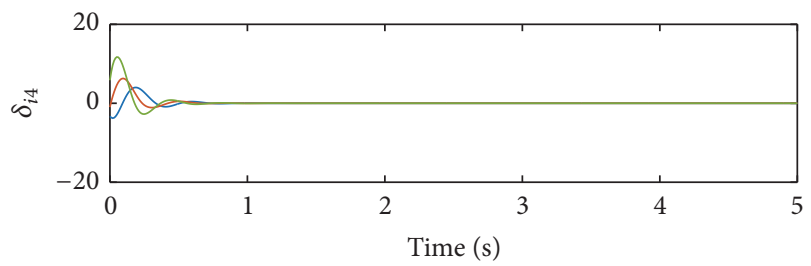

(d)

FIgure 6: Time evolution of consensus errors $\delta_{i}(t)=x_{i}(t)-$ $x_{1}(t)(i=2,3,4)$ in Example 2.

analyze the consensus behavior. By converting the consensus problem into the stability problem of a switched nonlinear system and constructing an appropriate multiple Lyapunov function, it has been proved that consensus behavior can be achieved under the proposed consensus protocols. By solving a Lyapunov equation and an algebraic Riccati equation, we provided a multistep algorithm to design the gain matrix in the proposed protocols. Overall, both theoretical analysis and numerical simulations show that the obtained results are quite efficient and could be further extended to solve other nonlinear MASs (e.g., QUAD nonlinear dynamics [38], unknown nonlinear dynamics [39]). Moreover, although this result hinges on deterministic switching topologies, the information flow between agents may change due to the link failure with a certain probability and the communication topologies in such cases will switch stochastically [40, 41]. Therefore, the consensus issue of high-order nonlinear MASs under random switching topologies deserves careful studies. Future efforts will focus on solving these problems. 


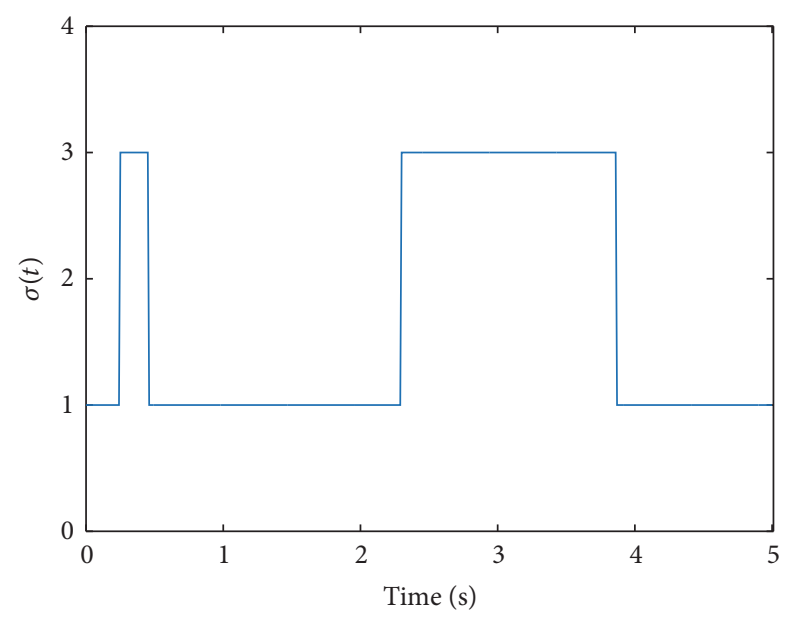

Figure 7: Time evolution of switching signal $\sigma(t)$ with $\tau_{a}=1.8$ in Example 2.

\section{Competing Interests}

The authors declare that they have no competing interests.

\section{Acknowledgments}

This work was jointly supported by the Characteristic Innovation Project of Education Department of Guangdong Province under Grant 2015KTSCX034, the Training Program for Outstanding Young Teachers in University of Guangdong Province under Grant Yq2013065, the China Postdoctoral Science Foundation under Grant 2013M540648, the Humanities and Social Science Research Foundation of Ministry of Education of China under Grant 15YJAZH051, and the Guangdong Natural Science Foundation under Grant 2015A030313574.

\section{References}

[1] J. G. Bender, "An overview of systems studies of automated highway systems," IEEE Transactions on Vehicular Technology, vol. 40, no. 1, pp. 82-99, 1991.

[2] C. Tomlin, G. J. Pappas, and S. Sastry, "Conflict resolution for air traffic management: a study in multiagent hybrid systems," IEEE Transactions on Automatic Control, vol. 43, no. 4, pp. 509521, 1998.

[3] R. W. Beard, T. W. McLain, M. A. Goodrich, and E. P. Anderson, "Coordinated target assignment and intercept for unmanned air vehicles," IEEE Transactions on Robotics and Automation, vol. 18, no. 6, pp. 911-922, 2002.

[4] A. Jadbabaie, J. Lin, and A. S. Morse, "Coordination of groups of mobile autonomous agents using nearest neighbor rules," IEEE Transactions on Automatic Control, vol. 48, no. 6, pp. 988-1001, 2003.

[5] R. Olfati-Saber and R. M. Murray, "Consensus problems in networks of agents with switching topology and time-delays," IEEE Transactions on Automatic Control, vol. 49, no. 9, pp. 15201533, 2004.
[6] L. Moreau, "Stability of multiagent systems with time-dependent communication links," IEEE Transactions on Automatic Control, vol. 50, no. 2, pp. 169-182, 2005.

[7] W. Ren and R. W. Beard, "Consensus seeking in multiagent systems under dynamically changing interaction topologies," IEEE Transactions on Automatic Control, vol. 50, no. 5, pp. 655661, 2005.

[8] W. Yu, G. Chen, and M. Cao, "Consensus in directed networks of agents with nonlinear dynamics," IEEE Transactions on Automatic Control, vol. 56, no. 6, pp. 1436-1441, 2011.

[9] Y. Cao and W. Ren, "Finite-time consensus for multi-agent networks with unknown inherent nonlinear dynamics," Automatica, vol. 50, no. 10, pp. 2648-2656, 2014.

[10] W. Ren, "On consensus algorithms for double-integrator dynamics," IEEE Transactions on Automatic Control, vol. 53, no. 6, pp. 1503-1509, 2008.

[11] K. Chen, J. Wang, Y. Zhang, and Z. Liu, "Consensus of secondorder nonlinear multi-agent systems under state-controlled switching topology," Nonlinear Dynamics, vol. 81, no. 4, pp. 1871-1878, 2015.

[12] L. Rong and H. Shen, "Distributed containment control of second-order multiagent systems with input delays under general protocols," Complexity, vol. 21, no. 6, pp. 112-120, 2016.

[13] L. Rong, H. Shen, and J. Li, "Distributed quantised consensus in groups of agents with acceleration-like inputs: a reference model-based scheme," IET Control Theory \& Applications, vol. 10, no. 5, pp. 590-598, 2016.

[14] P. Lin, Z. Li, Y. Jia, and M. Sun, "High-order multi-agent consensus with dynamically changing topologies and timedelays," IET Control Theory \& Applications, vol. 5, no. 8, pp. 976981, 2011.

[15] Y. Cao, W. Yu, W. Ren, and G. Chen, "An overview of recent progress in the study of distributed multi-agent coordination," IEEE Transactions on Industrial Informatics, vol. 9, no. 1, pp. 427-438, 2013.

[16] Z. Li, Z. Duan, G. Chen, and L. Huang, "Consensus of multiagent systems and synchronization of complex networks: a unified viewpoint," IEEE Transactions on Circuits and Systems. I. Regular Papers, vol. 57, no. 1, pp. 213-224, 2010.

[17] C.-Q. Ma and J.-F. Zhang, "Necessary and sufficient conditions for consensusability of linear multi-agent systems," IEEE Transactions on Automatic Control, vol. 55, no. 5, pp. 1263-1268, 2010.

[18] H. Zhao and J. H. Park, "Dynamic output feedback consensus of continuous-time networked multiagent systems," Complexity, vol. 20, no. 5, pp. 35-42, 2015.

[19] S. E. Tuna, "Conditions for synchronizability in arrays of coupled linear systems," IEEE Transactions on Automatic Control, vol. 54, no. 10, pp. 2416-2420, 2009.

[20] I. Saboori and K. Khorasani, "H1 consensus achievement of multi-agent systems with directed and switching topology networks," IEEE Transactions on Automatic Control, vol. 59, no. 11, pp. 3104-3109, 2014.

[21] W. Ni and D. Cheng, "Leader-following consensus of multiagent systems under fixed and switching topologies," Systems \& Control Letters, vol. 59, no. 3-4, pp. 209-217, 2010.

[22] J. Qin, C. Yu, and H. Gao, "Coordination for linear multiagent systems with dynamic interaction topology in the leaderfollowing framework," IEEE Transactions on Industrial Electronics, vol. 61, no. 5, pp. 2412-2422, 2014.

[23] Z. Li, W. Ren, X. Liu, and M. Fu, "Consensus of multi-agent systems with general linear and Lipschitz nonlinear dynamics 
using distributed adaptive protocols," IEEE Transactions on Automatic Control, vol. 58, no. 7, pp. 1786-1791, 2013.

[24] Z. Peng, D. Wang, H. Zhang, and G. Sun, "Distributed neural network control for adaptive synchronization of uncertain dynamical multiagent systems," IEEE Transactions on Neural Networks and Learning Systems, vol. 25, no. 8, pp. 1508-1519, 2014.

[25] B. Kaviarasan, R. Sakthivel, and S. Abbas, "Robust consensus of nonlinear multi-agent systems via reliable control with probabilistic time delay," Complexity, 2016.

[26] Q. Jia and W. K. Tang, "Consensus of nonlinear agents in directed network with switching topology and communication delay," IEEE Transactions on Circuits and Systems. I. Regular Papers, vol. 59, no. 12, pp. 3015-3023, 2012.

[27] G. Wen, Z. Duan, G. Chen, and W. Yu, "Consensus tracking of multi-agent systems with Lipschitz-type node dynamics and switching topologies," IEEE Transactions on Circuits and Systems I: Regular Papers, vol. 61, no. 2, pp. 499-511, 2014.

[28] W. Xu, J. Cao, W. Yu, and J. Lu, "Leader-following consensus of non-linear multi-agent systems with jointly connected topology," IET Control Theory \& Applications, vol. 8, no. 6, pp. 432440, 2014.

[29] W. Liu, S. Zhou, Y. Qi, and X. Wu, "Leaderless consensus of multi-agent systems with Lipschitz nonlinear dynamics and switching topologies," Neurocomputing, vol. 173, pp. 1322-1329, 2016.

[30] C. Godsil and G. Royle, Algebraic Graph Theory, Springer, New York, NY, USA, 2001.

[31] Y. G. Sun and L. Wang, "Consensus of multi-agent systems in directed networks with nonuniform time-varying delays," IEEE Transactions on Automatic Control, vol. 54, no. 7, pp. 1607-1613, 2009.

[32] A. J. Laub, Matrix Analysis for Scientists and Engineers, SIAM, Philadelphia, Pa, USA, 2005.

[33] E. D. Sontag, Mathematical Control Theory: Deterministic Finite Dimensional Systems, Springer, New York, NY, USA, 1998.

[34] J. P. Hespanha and A. S. Morse, "Stability of switched systems with average dwell-time," in Proceedings of the 38th IEEE Conference on Decision and Control (CDC '99), pp. 2655-2660, Phoenix, Ariz, USA, December 1999.

[35] T. Matsumoto, L. O. Chua, and K. Ayaki, "Reality of chaos in the double scroll circuit: a computer-assisted proof," IEEE Transactions on Circuits and Systems, vol. 35, no. 7, pp. 909-925, 1988.

[36] X. Liu and T. Chen, "Exponential synchronization of nonlinear coupled dynamical networks with a delayed coupling," Physica A, vol. 381, no. 1-2, pp. 82-92, 2007.

[37] R. Rajamani and Y. M. Cho, "Existence and design of observers for nonlinear systems: relation to distance to unobservability," International Journal of Control, vol. 69, no. 5, pp. 717-731, 1998.

[38] P. DeLellis, M. di Bernardo, and G. Russo, "On QUAD, Lipschitz, and contracting vector fields for consensus and synchronization of networks," IEEE Transactions on Circuits and Systems I: Regular Papers, vol. 58, no. 3, pp. 576-583, 2011.

[39] H. W. Zhang and F. L. Lewis, "Adaptive cooperative tracking control of higher-order nonlinear systems with unknown dynamics," Automatica, vol. 48, no. 7, pp. 1432-1439, 2012.

[40] M. Porfiri and D. J. Stilwell, "Consensus seeking over random weighted directed graphs," IEEE Transactions on Automatic Control, vol. 52, no. 9, pp. 1767-1773, 2007.
[41] M. Porfiri, D. J. Stilwell, and E. M. Bollt, "Synchronization in random weighted directed networks," IEEE Transactions on Circuits and Systems I: Regular Papers, vol. 55, no. 10, pp. 31703177, 2008. 


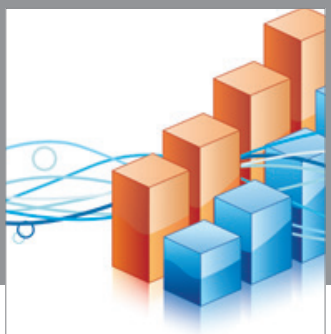

Advances in

Operations Research

vatem alat4

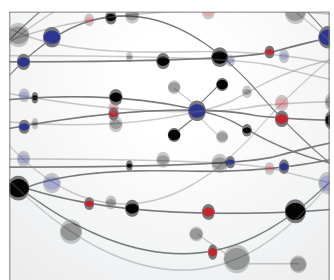

\section{The Scientific} World Journal
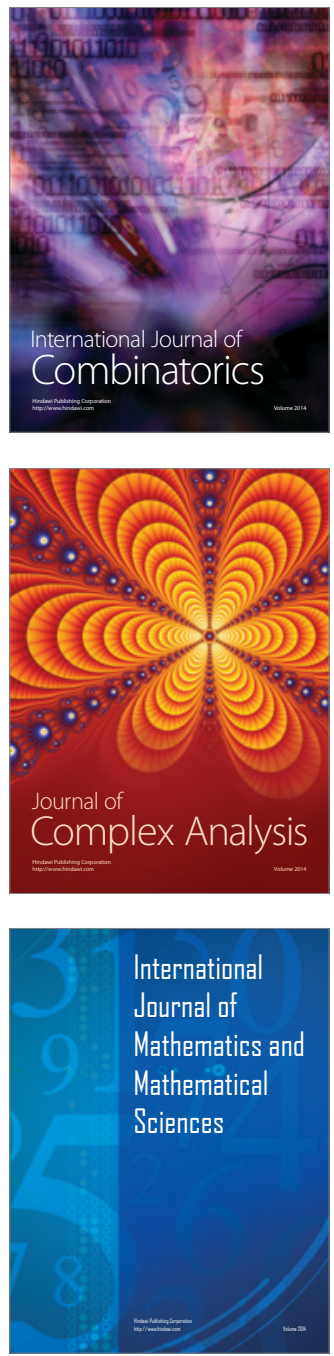
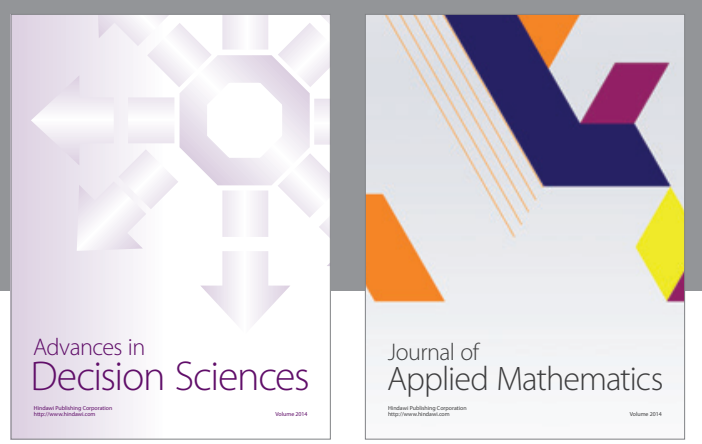

Algebra

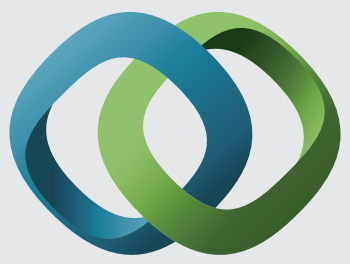

\section{Hindawi}

Submit your manuscripts at

https://www.hindawi.com
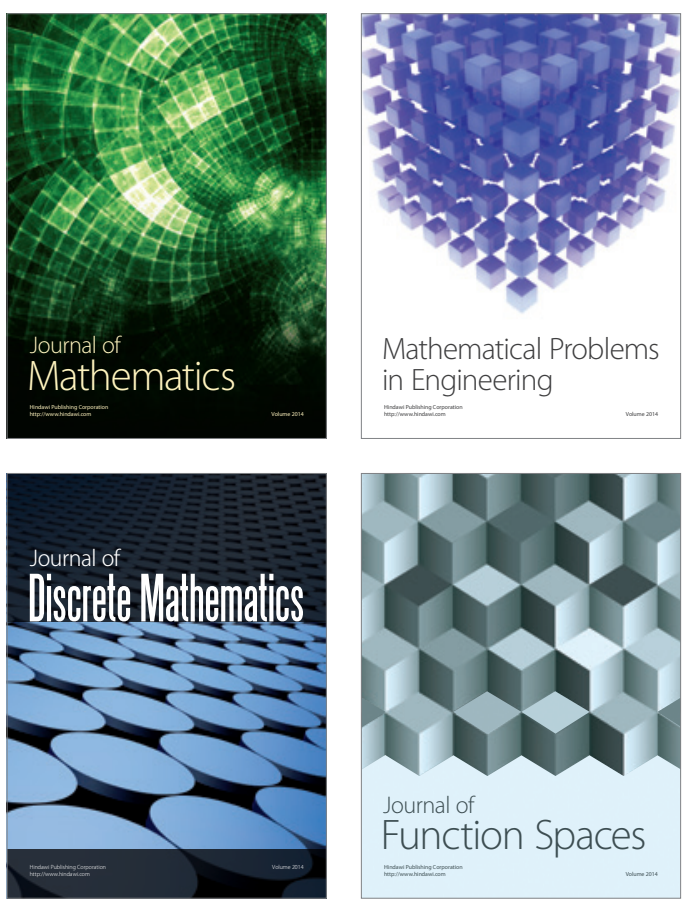

Mathematical Problems in Engineering
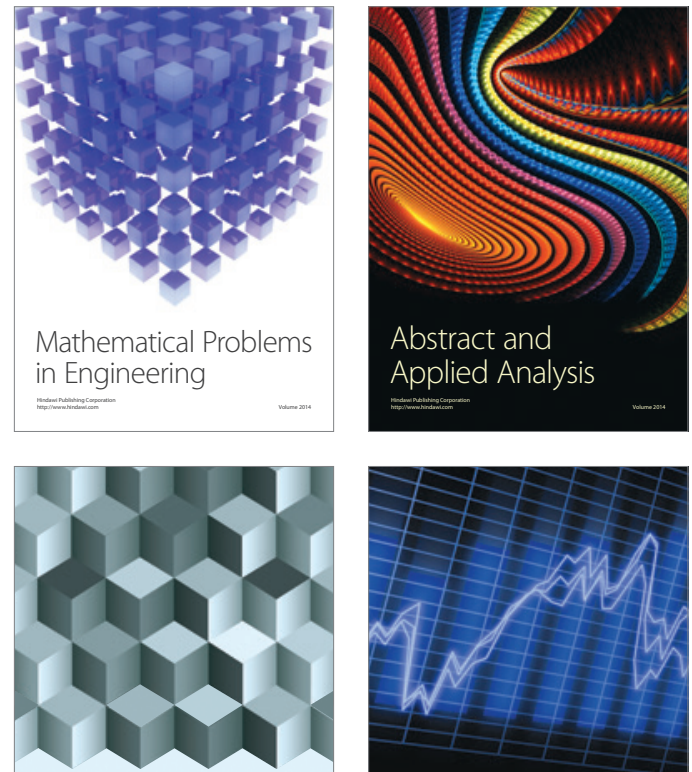

Journal of

Function Spaces

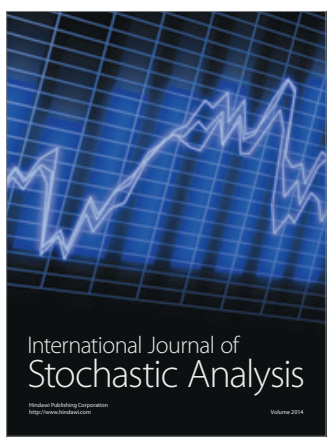

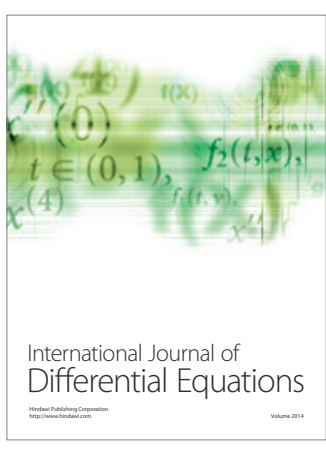
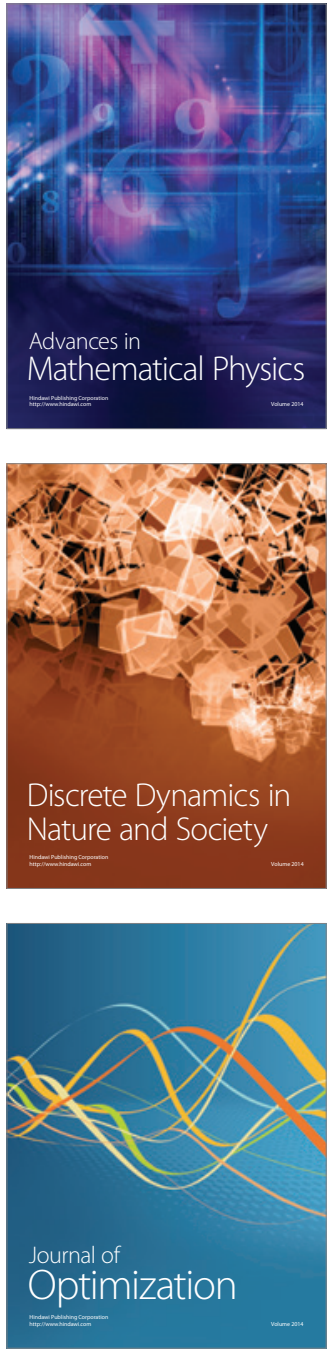